\title{
PAPER \\ Dominating Sets and Induced Matchings in Orthogonal Ray Graphs
}

\author{
Asahi TAKAOKA ${ }^{\dagger a)}$, Student Member, Satoshi TAYU ${ }^{\dagger b)}$, Member, and Shuichi UENO $^{\dagger c)}$, Fellow
}

\begin{abstract}
SUMMARY An orthogonal ray graph is an intersection graph of horizontal and vertical rays (closed half-lines) in the plane. Such a graph is 3 -directional if every vertical ray has the same direction, and 2-directional if every vertical ray has the same direction and every horizontal ray has the same direction. We derive some structural properties of orthogonal ray graphs, and based on these properties, we introduce polynomial-time algorithms that solve the dominating set problem, the induced matching problem, and the strong edge coloring problem for these graphs. We show that for 2-directional orthogonal ray graphs, the dominating set problem can be solved in $O\left(n^{2} \log ^{5} n\right)$ time, the weighted dominating set problem can be solved in $O\left(n^{4} \log n\right)$ time, and the number of dominating sets of a fixed size can be computed in $O\left(n^{6} \log n\right)$ time, where $n$ is the number of vertices in the graph. We also show that for 2-directional orthogonal ray graphs, the weighted induced matching problem and the strong edge coloring problem can be solved in $O\left(n^{2}+m \log n\right)$ time, where $m$ is the number of edges in the graph. Moreover, we show that for 3-directional orthogonal ray graphs, the induced matching problem can be solved in $O\left(\mathrm{~m}^{2}\right)$ time, the weighted induced matching problem can be solved in $O\left(\mathrm{~m}^{4}\right)$ time, and the strong edge coloring problem can be solved in $O\left(\mathrm{~m}^{3}\right)$ time. We finally show that the weighted induced matching problem can be solved in $O\left(\mathrm{~m}^{6}\right)$ time for orthogonal ray graphs.

key words: boolean-width, dominating set, induced matching, orthogonal ray graphs, strong edge coloring
\end{abstract}

\section{Introduction}

A bipartite graph $G$ with bipartition $(U, V)$ is called an orthogonal ray graph (ORG for short) [50] if there exist a set of disjoint horizontal rays (closed half-lines) $R_{u}, u \in U$, in the $x y$-plane and a set of disjoint vertical rays $R_{v}, v \in V$, such that for any $u \in U$ and $v \in V,(u, v) \in E(G)$ if and only if $R_{u}$ and $R_{v}$ intersect. Set $\mathcal{R}(G)=\left\{R_{w} \mid w \in V(G)\right\}$ is called the orthogonal ray representation of $G$. ORGs are introduced in connection with the defect-tolerant design of nanocircuits [49]. An ORG is called a 3-directional orthogonal ray graph (3-DORG for short) if every vertical ray $R_{v}, v \in V$, has the same direction. A 3-ORG is called a 2-directional orthogonal ray graph (2-DORG for short) if every horizontal ray $R_{u}, u \in U$, has the same direction. We also refer to such representation of 3- and 2-DORGs as the orthogonal ray representation.

The following relationship between bipartite graph

Manuscript received June 5, 2014.

Manuscript revised September 1, 2014.

Manuscript publicized September 9, 2014.

${ }^{\dagger}$ The authors are with the Department of Communications and Computer Engineering, Tokyo Institute of Technology, Tokyo, 152-8550 Japan.

a)E-mail: asahi@eda.ce.titech.ac.jp

b)E-mail: tayu@eda.ce.titech.ac.jp

c) E-mail: ueno@eda.ce.titech.ac.jp

DOI: $10.1587 /$ transinf.2014EDP7184 classes is known [44], [50]: \{Bipartite Permutation Graphs\} $\subset\{$ Biconvex Graphs $\} \subset\{$ Convex Graphs $\} \subset\{$ Interval Bigraphs $\} \subset\{2$-Directional Orthogonal Ray Graphs $\} \subset$ \{Chordal Bipartite Graphs\}, and \{2-Directional Orthogonal Ray Graphs $\} \subset\{$ 3-Directional Orthogonal Ray Graphs $\} \subset$ $\{$ Orthogonal Ray Graphs\} $\subset$ \{Unit Grid Intersection Graphs\} $\subset\{$ Grid Intersection Graphs\}, where $X \subset Y$ indicates a set $X$ is a proper subset of $Y$. Definitions and some comprehensive surveys of these graphs can be found in [10], [30], [50], [53].

The class of 2-DORGs has been well studied, and various characterizations are known [50], [52]. Based on a characterization by circular-arc graphs, 2-DORGs can be recognized in $O\left(n^{2}\right)$ time, and the orthogonal ray representation of a 2-DORG can be obtained in $O\left(n^{2}\right)$ time [50], where $n$ is the number of vertices in the graph. It is known that the biclique problem can be solved in $O(n)$ time provided that orthogonal ray representations are given [49]. It is also known that the cross-free matching problem, the biclique cover problem, and the jump number problem can be solved in $O\left(n^{2.5} \log ^{c} n\right)$ time for some constant $c$ [52]. Based on a characterization by vertex ordering, the matching problem and the oriented coloring problem can be solved in linear time [22]. Moreover, it is known that the node-weighted matching problem can be solved in $O\left(n \log ^{2} n\right)$ time provided that orthogonal ray representations are given [46].

This paper considers the complexities of the dominating set problem, the induced matching problem, and the strong edge coloring problem for ORGs. We introduce the first polynomial-time algorithms to solve the problems for 2-DORGs, 3-DORGs, or ORGs. We also show faster algorithms to solve some problems for 2-DORGs.

\subsection{Dominating Set Problem}

Vertex set $D \subseteq V(G)$ is called a dominating set of a graph $G$ if for any $v \in V(G) \backslash D$, there exists a vertex in $D$ adjacent to $v$. The (weighted) dominating set problem is to find a dominating set with minimum cardinality (weight) in a given (vertex-weighted) graph. The dominating set problem is one of the most basic and well-studied problems in graph algorithms. The problem and its variants have many applications in various areas including computer networks, and have been received considerable attention. Also, the dominating set counting problem in graph classes is considered [37].

The dominating set problem is NP-hard for gen- 
eral graphs [29], and remains NP-hard for chordal bipartite graphs [43] and for unit grid intersection graphs [45], whereas the problem can be solved in $O(n)$ time for permutation graphs [19] and in $O\left(n^{3}\right)$ time for convex graphs [21]. Also, the weighted dominating set problem can be solved in $O(n+m)$ time for permutation graphs [47] and in polynomial time for convex graphs [28] and interval bigraphs [35], where $m$ is the number of edges in the graph. Moreover, counting the number of dominating sets in a chordal bipartite graph is known to be $\sharp \mathrm{P}$-complete [37].

We consider in Sect. 3 the complexity of the (weighted) dominating set problem for (vertex-weighted) 2-DORGs and the dominating set counting problem. We show in Sect. 3.1 that the dominating set problem can be solved in $O\left(n^{2} \log ^{5} n\right)$ time for 2-DORGs, by showing that 2-DORGs are the complements of bounded tolerance graphs. The weighted dominating set problem and the dominating set counting problem for ORGs are recently pointed out to be solvable in polynomial time [56] provided that orthogonal ray representations are given, by using a new parameter, boolean-width of graphs, which is introduced in [12], [13]. We show in Sect. 3.2 that, indeed, the weighted dominating set problem can be solved in $O\left(n^{4} \log n\right)$ time for vertexweighted 2-DORGs, and the number of dominating sets in a 2-DORG of a fixed size can be computed in $O\left(n^{6} \log n\right)$ time. The results above are in contrast with the NP-hardness of the dominating set problem [43] and the $\sharp P$-completeness of the dominating set counting problem [37] for chordal bipartite graphs. Thus, the class of 2-DORGs is a boundary for the complexities of these problems.

\subsection{Induced Matching and Strong Edge Coloring Problem}

A matching of a graph $G$ is a set of edges no two of which share a common vertex in $G$. An induced matching of $G$ is a matching that is also an induced subgraph. The (weighted) induced matching problem is to find an induced matching with maximum cardinality (weight) in a given (edgeweighted) graph. Recently, the induced matching problem has attracted much attention. The problem has many applications such as the risk-free marriage [54] and the secure messages broadcast on communication channels [32]. It also has the applications for wireless networks: The maximum induced matchings are used to model a kind of capacity of wireless networks [2], and the problem is used to show the NP-hardness of the wireless gathering problem [7], [42].

The induced matching problem is NP-hard for general bipartite graphs [15], [54], and even for unit grid intersection graphs [45], whereas the problem can be solved in $O(n+m)$ time for bipartite permutation graphs [18], in $O\left(n^{2}\right)$ time for convex graphs [8], in $O(\mathrm{~nm})$ time for interval bigraphs [1], and in $O\left(\mathrm{~m}^{3}\right)$ time for chordal bipartite graphs [1], [17], [22]. Also, the weighted induced matching problem can be solved in $O\left(\mathrm{~m}^{4}\right)$ time for chordal bipartite graphs [17]. Since the class of 2-DORGs is contained in the class of chordal bipartite graphs, it has been already known that the induced matching problem for 2-DORGs can be solved in $O\left(\mathrm{~m}^{3}\right)$ time, and $O\left(\mathrm{~m}^{4}\right)$ time for weighted case.

A strong edge coloring of a graph $G$ is an assignment of colors to the edges of $G$ such that every two edges with adjacent endvertices must have different colors. In other words, a strong edge coloring of $G$ is a partition of $E(G)$ into a collection of induced matchings. The strong edge coloring problem is to find a strong edge coloring with the minimum number of colors. The problem has applications for wireless networks [3], and has been well studied (see [40] for survey).

We consider in Sect. 4 the complexity of the (weighted) induced matching problem and the strong edge coloring problem for ORGs. We show in Sect. 4.1 that for 2-DORGs, the weighted induced matching problem and the strong edge coloring problem can be solved in $O\left(n^{2}+m \log n\right)$ time by reducing the problems to the weighted independent set problem and the coloring problem for trapezoid graphs, respectively. It should be noted that $O\left(n^{2}\right)$ time is needed to obtain the orthogonal ray representation of a graph. This reduce the complexity of the (weighted) induced matching problem for 2-DORGs. We also show in Sect. 4.2 that for 3-DORGs, the induced matching problem can be solved in $O\left(\mathrm{~m}^{2}\right)$ time, the weighted induced matching problem can be solved in $O\left(\mathrm{~m}^{4}\right)$ time, and the strong edge coloring problem can be solved in $O\left(\mathrm{~m}^{3}\right)$ time by reducing the problems to the (weighted) independent set problem and the coloring problem for cocomparability graphs, respectively. Moreover, we show in Sect. 4.3 that the weighted induced matching problem can be solved in $O\left(\mathrm{~m}^{6}\right)$ time for ORGs. This is in contrast with the NP-hardness of the problem for unit grid intersection graphs, and thus, the class of ORGs is a boundary for the complexity of the induced matching problem.

\section{Preliminaries}

All graphs considered in this paper are finite, simple, and undirected. For a graph $G$, let $V(G)$ and $E(G)$ denote the set of vertices and edges, respectively. Let $n=|V(G)|$ and $m=|E(G)|$. The complement of $G$ is the graph $\bar{G}$ such that $V(\bar{G})=V(G)$ and $E(\bar{G})=\{(u, v) \mid(u, v) \notin E(G)\}$. The open neighborhood of a vertex $v$ in $G$ is the set $N_{G}(v)=\{u \in$ $V(G) \mid(u, v) \in E(G)\}$, and the closed neighborhood of a vertex $v$ in $G$ is the set $N_{G}[v]=\{v\} \cup N_{G}(v)$. For an edge $e=(u, v) \in E(G)$, we use $N_{G}[e]$ to denote the set of vertices adjacent to $u$ or $v$, that is,

$$
N_{G}[e] \stackrel{\text { def }}{=} N_{G}[u] \cup N_{G}[v] \text { for an edge } e=(u, v) .
$$

If no confusion arise, we will omit the index.

A point $p=(x, y)$ in the $x y$-plane is said to be dominated by a point $p^{\prime}=\left(x^{\prime}, y^{\prime}\right)$, denoted by $p<\mathbb{R}^{2} p^{\prime}$, if $x<x^{\prime}$ and $y<y^{\prime}$. It is shown in [51], [52] that a bipartite graph $G$ with bipartition $(U, V)$ is a 2-DORG if and only if there exists a set of points $p_{w}, w \in V(G)$, in the $x y$-plane such that for any $u \in U$ and $v \in V,(u, v) \in E(G)$ if and only if $p_{u}<\mathbb{R}^{2} p_{v}$. Moreover, we can assume without loss of generality that every $x_{w}$ is distinct and every $y_{w}$ is distinct. A set 
of points $\mathcal{P}(G)=\left\{p_{w} \mid w \in V(G)\right\}$ is called the point representation of $G$. The point representation can be obtained from the orthogonal ray representation as follows: Consider the orthogonal ray representation of $G$ consisting of rightward rays corresponding to the vertices of $U$ and downward rays corresponding to the vertices of $V$. The set of endpoints of rays is the point representation of $G$.

Let $L_{1}$ and $L_{2}$ be two lines parallel to $x$-axis in the $x y$ plane. A graph $G$ is called a trapezoid graph [20] if there exists a set of trapezoids $T_{v}, v \in V(G)$, with parallel sides along $L_{1}$ and $L_{2}$ such that for any $u, v \in V(G),(u, v) \in E(G)$ if and only if $T_{u}$ and $T_{v}$ intersect. A trapezoid graph $G$ is called a parallelogram graph [6] if $T_{v}$ is a parallelogram for every $v \in V(G)$.

In stead of the definitions above, we use the characterizations of trapezoid graphs and parallelogram graphs that are shown in [24], [26]. An axis-parallel rectangle in the $x y$ plane is called a box. Let $\operatorname{ur}(B)$ denote the upper right corner point of a box $B$, and let $l l(B)$ denote the lower left corner point of $B$. A box $B$ is said to be dominated by a box $B^{\prime}$, denoted by $B<\mathbb{R}^{2} B^{\prime}$, if $\operatorname{ur}(B)<\mathbb{R}^{2} l l\left(B^{\prime}\right)$. It is shown in [26] that a graph $G$ is a trapezoid graph if and only if there exists a set of boxes $B_{v}, v \in V(G)$, in the $x y$-plane such that for any $u, v \in V(G),(u, v) \in E(G)$ if and only if $B_{u} \nless_{\mathbb{R}^{2}} B_{v}$ and $B_{v} \nless_{\mathbb{R}^{2}} B_{u}$. A set of boxes $\mathcal{B}(G)=\left\{B_{v} \mid v \in V(G)\right\}$ is called the box representation of $G$. It is also shown in [24] that a trapezoid graph $G$ is a parallelogram graph if and only if $B_{v}$ is a square for every $v \in V(G)$.

A graph $G$ is called a circular-arc graph if there exists a set of arcs $A_{v}, v \in V(G)$, on a circle such that for any $u, v \in V(G),(u, v) \in E(G)$ if and only if $A_{u}$ and $A_{v}$ intersect.

A bipartite graph $G$ with bipartition $(U, V)$ is called an interval containment bigraph if there exists a set of intervals $I_{w}, w \in V(G)$, on the real line such that for any $u \in U$ and $v \in V,(u, v) \in E(G)$ if and only if $I_{u} \subseteq I_{v}$.

A graph $G$ is called a tolerance graph if there exist a set of intervals $I_{v}, v \in V(G)$, on the real line and a set of positive numbers $t_{v}, v \in V(G)$, called tolerances, such that for any $u, v \in V(G),(u, v) \in E(G)$ if and only if $\left|I_{u} \cap I_{v}\right| \geq \min \left\{t_{u}, t_{v}\right\}$, where $|I|$ is the length of $I$. A tolerance graph $G$ is called a bounded tolerance graph if $t_{v} \leq\left|I_{v}\right|$ for every $v \in V(G)$.

A pair $P=(A,<)$ of a set $A$ and a reflexive, antisymmetric, and transitive binary relation $<$ on $A$ is called a partially ordered set (poset for short). A graph $G$ is called a comparability graph of a poset $P$ if there exists a bijection assigning each $v \in V(G)$ to $a_{v} \in A$ such that for any $u, v \in V(G),(u, v) \in E(G)$ if and only if $a_{u}$ and $a_{v}$ are comparable in $P$, that is, $a_{u}<a_{v}$ or $a_{v}<a_{u}$. A graph $G$ is called a co-comparability graph if $\bar{G}$ is a comparability graph.

A poset $P=(A,<)$ is called an interval order if there exists a set of intervals $I_{a}, a \in A$, on the real line such that for any $a, b \in A, a<b$ if and only if $r_{a}<l_{b}$, where $r_{a}$ is the right endpoint of $I_{a}$ and $l_{b}$ is the left endpoint of $I_{b}$. The interval dimension of a poset $P$ is the minimum number $d$ of interval orders $P_{i}=\left(A,<_{i}\right), i \in\{1,2, \ldots, d\}$, such that for any $a, b \in A, a<b$ if and only if $a<_{i} b$ for every $i \in$ $\{1,2, \ldots, d\}$. A poset is said to be height 1 if the underlying

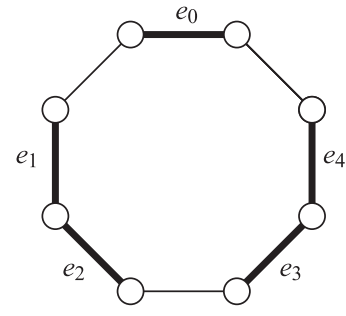

Fig. 1 An example of edge-asteroid of size 5.

graph is bipartite.

A sequence of vertices $\left(v_{0}, v_{1}, \ldots, v_{2 k}\right), k \geq 1$, of a graph $G$ is called an asteroid of size $2 k+1$ if for any $i, 0 \leq i \leq 2 k$, there exists a path from $v_{i}$ to $v_{i+1}$ that contains no vertices in $N\left[v_{i+k+1}\right]$ (Subscripts are modulo $2 k+1$ ). The following is shown in [27].

Theorem I. A graph is a co-comparability graph if and only if it contains no asteroids.

A bipartite graph is called a chordal bipartite graph if it contains no cycle of length at least 6 as an induced subgraph.

A sequence of edges $\left(e_{0}, e_{1}, \ldots, e_{2 k}\right), k \geq 1$, of a graph $G$ is called an edge-asteroid of size $2 k+1$ if for any $i, 0 \leq$ $i \leq 2 k$, there exists a path from $e_{i}$ to $e_{i+1}$ that contains no vertices in $N\left[e_{i+k+1}\right]$ (Subscripts are modulo $2 k+1$ ). See Fig. 1 for an example. Edge-asteroids are introduced in [23], and 2-DORGs can be characterized as follows [23], [50].

Theorem II. A bipartite graph is a 2-DORG if and only if it is a chordal bipartite graph and contains no edge-asteroids.

A bipartite graph $G$ with bipartition $(U, V)$ is called a chain graph if for any pair of vertices $u, u^{\prime} \in U$, either $N(u) \subseteq N\left(u^{\prime}\right)$ or $N(u) \supseteq N\left(u^{\prime}\right)$ (it is also known as a difference graph [33] and a Ferrers bigraph [34]). A disconnected graph consisting of $k$ disjoint edges is denoted by $k K_{2}$. It is known that a bipartite graph is a chain graph if and only if it contains no $2 K_{2}$ as an induced subgraph [58], and thus, the size of the maximum induced matching of any chain graph is at most 1. A graph $G$ is called the union of graphs $G_{i}, 1 \leq i \leq k$, if $E(G)=E\left(G_{1}\right) \cup E\left(G_{2}\right) \cup \cdots \cup E\left(G_{k}\right)$, and called the intersection of graphs $G_{i}, 1 \leq i \leq k$, if $E(G)=$ $E\left(G_{1}\right) \cap E\left(G_{2}\right) \cap \cdots \cap E\left(G_{k}\right)$. The chain subgraph cover problem for a bipartite graph $G$ is to find the minimum number of chain graphs, denoted by $\operatorname{ch}(G)$, whose union is $G$. It is known that for any chordal bipartite graph $G$, the size of the maximum induced matching of $G$ is equal to $\operatorname{ch}(G)$ [1]. The chain dimension of a bipartite graph $G$, denoted by $\operatorname{cdim}(G)$, is the minimum number of chain graphs whose intersection is $G$ (The chain dimension is also known as the Ferrers dimension [34]). It is known that for any fixed $k \geq 3$, deciding whether $\operatorname{ch}(G) \leq k$ for a given bipartite graph $G$ and deciding whether $\operatorname{cdim}(G) \leq k$ are NP-complete [58], while deciding whether $\operatorname{ch}(G) \leq 2$ and deciding whether $\operatorname{cdim}(G) \leq 2$ can be solved in $O\left(n^{2}\right)$ time [39].

Some comprehensive surveys with other results can be 
found in [10], [30], [53].

\section{Dominating Sets in 2-DORGs}

\subsection{Dominating Set Problem}

The following relationships are known:

- A bipartite graph $G$ is a 2-DORG if and only if $\bar{G}$ is a circular-arc graph [50];

- A bipartite graph $G$ is an interval containment bigraph if and only if $\operatorname{cdim}(G) \leq 2$, and if and only if $\bar{G}$ is a circular-arc graph [34];

- A bipartite graph $G$ is a comparability graph of a poset of interval dimension at most 2 and height 1 if and only if $\bar{G}$ is a circular-arc graph [55];

- A graph $G$ is a comparability graph of a poset of interval dimension at most 2 if and only if $\bar{G}$ is a trapezoid graph [20];

- A graph $G$ is a parallelogram graph if and only if $G$ is a bounded tolerance graph [6], [24].

We list the graph classes equivalent to 2-DORGs including the classes above.

Theorem 1. The following conditions are equivalent for a bipartite graph $G$ :

(i) $G$ is a 2-directional orthogonal ray graph;

(ii) $G$ is an interval containment bigraph;

(iii) $G$ is a bipartite graph with chain dimension at most 2 ;

(iv) $G$ is a comparability graph of a poset of interval dimension at most 2 and height 1 ;

(v) $\bar{G}$ is a circular-arc graph;

(vi) $\bar{G}$ is a trapezoid graph;

(vii) $\bar{G}$ is a parallelogram graph;

(viii) $\bar{G}$ is a bounded tolerance graph.

Proof. Since the equivalences (i) $\Leftrightarrow$ (ii) $\Leftrightarrow$ (iii) $\Leftrightarrow$ (iv) $\Leftrightarrow$ (v) $\Leftrightarrow$ (vi) are shown in [20], [34], [50], [55], and the equivalence (vii) $\Leftrightarrow$ (viii) is shown in [6], [24], it suffices to show the equivalence (i) $\Leftrightarrow$ (vii).

Proof of (i) $\Rightarrow$ (vii). Let $G$ be a 2-DORG with bipartition $(U, V)$ and a point representation $\mathcal{P}(G)=\left\{\left(x_{w}, y_{w}\right) \mid\right.$ $w \in V(G)\}$. We assume without loss of generality that every $x_{w}$ is distinct and every $y_{w}$ is distinct [52]. For each $u \in U$, let $B_{u}$ be a square with $\operatorname{ur}\left(B_{u}\right)=\left(x_{u}, y_{u}\right)$ and $l l\left(B_{u}\right)=$ $\left(x_{u}-s, y_{u}-s\right)$, where $s>\max _{u, u^{\prime} \in U}\left\{\left|x_{u}-x_{u^{\prime}}\right|,\left|y_{u}-y_{u^{\prime}}\right|\right\}$ (See Fig. 2 for example). For each $v \in V$, let $B_{v}$ be a square with $\operatorname{ur}\left(B_{v}\right)=\left(x_{v}+t, y_{v}+t\right)$ and $\operatorname{ll}\left(B_{v}\right)=\left(x_{v}, y_{v}\right)$, where $t>\max _{v, v^{\prime} \in V}\left\{\left|x_{v}-x_{v^{\prime}}\right|,\left|y_{v}-y_{v^{\prime}}\right|\right\}$. Let $H$ be a parallelogram graph with $V(H)=V(G)$ and the box representation $\mathcal{B}(H)=\left\{B_{w} \mid w \in V(G)\right\}$. Notice that $\bar{H}$ is a bipartite graph with bipartition $(U, V)$ since $s$ and $t$ are sufficiently large. Since $(u, v) \in E(G) \Leftrightarrow\left(x_{u}, y_{u}\right)<\mathbb{R}^{2}\left(x_{v}, y_{v}\right) \Leftrightarrow B_{u}<\mathbb{R}^{2} B_{v} \Leftrightarrow$ $(u, v) \notin E(H) \Leftrightarrow(u, v) \in E(\bar{H})$, we conclude that $H=\bar{G}$. Thus, $\bar{G}$ is a parallelogram graph.

Proof of (i) $\Leftarrow$ (vii). Let $G$ be a bipartite graph with

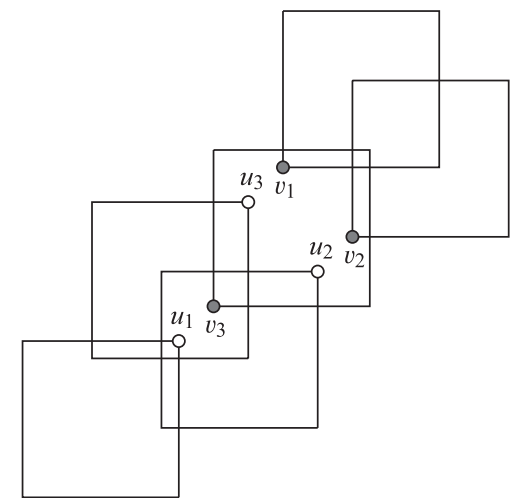

Fig. 2 The box representation of a 2-directional orthogonal ray graph constructed from the point representation of the graph.

bipartition $(U, V)$ such that $\bar{G}$ is a parallelogram graph with a box representation $\mathcal{B}(\bar{G})=\left\{B_{w} \mid w \in V(G)\right\}$. For each $(u, v) \notin E(\bar{G})$, there exist squares $B_{u}$ and $B_{v}$ such that $B_{u}<\mathbb{R}^{2}$ $B_{v}$ or $B_{v}<\mathbb{R}^{2} B_{u}$. We can assume without loss of generality that $B_{u}<\mathbb{R}^{2} B_{v}$ for every $u \in U$ and $v \in V$. For each $u \in U$, let $\left(x_{u}, y_{u}\right)=u r\left(B_{u}\right)$, and for each $v \in V$, let $\left(x_{v}, y_{v}\right)=l l\left(B_{v}\right)$. We can further assume that every $x_{w}$ is distinct and every $y_{w}$ is distinct [36]. Let $H$ be a 2-DORG with $V(H)=V(G)$ and the point representation $\mathcal{P}(H)=\left\{\left(x_{w}, y_{w}\right) \mid w \in V(G)\right\}$. Since $(u, v) \notin E(\bar{G}) \Leftrightarrow B_{u}<\mathbb{R}^{2} \quad B_{v} \Leftrightarrow\left(x_{u}, y_{u}\right)<\mathbb{R}^{2}\left(x_{v}, y_{v}\right)$ $\Leftrightarrow(u, v) \in E(H)$, we conclude that $H=G$. Thus, $G$ is a 2-DORG.

It is shown in [36] that the dominating set problem can be solved in $O\left(n^{2} \log ^{5} n\right)$ time for the complements of parallelogram graphs provided that box representations are given, while the problem is NP-hard for the complements of trapezoid graphs. As shown in the proof of Theorem 1, the box representation of a 2-DORG $G$ can be obtained from the point representation of $G$. Since the point representation of $G$ can be obtained in $O\left(n^{2}\right)$ time [50], we have the following.

Theorem 2. The dominating set problem can be solved in $O\left(n^{2} \log ^{5} n\right)$ time for 2-DORGs.

\subsection{Weighted Dominating Set Problem and Dominating} Set Counting Problem

For a set of vertices $A \subseteq V(G)$ of a graph $G$, let $\bar{A}=V(G) \backslash A$. A bipartition $(A, \bar{A})$ of $V(G)$ is called a cut of $G$. Let the neighborhood of $A \subseteq V(G)$ be $N(A)=\bigcup_{v \in A} N(v)$. For a cut $(A, \bar{A})$ of $G$ and a subset $S \subseteq A$, the set $N(S) \cap \bar{A}$ is called the neighborhood of $S$ across $(A, \bar{A})$. Two subset $S, S^{\prime} \subseteq A$ are said to be neighborhood equivalent with respect to $A$, denoted by $S \equiv_{A} S^{\prime}$, if $S$ and $S^{\prime}$ have the same neighborhood across $(A, \bar{A})$, that is,

$$
S \equiv_{A} S^{\prime} \stackrel{\text { def }}{\Longleftrightarrow} N(S) \cap \bar{A}=N\left(S^{\prime}\right) \cap \bar{A} .
$$

The number of equivalence classes of $\equiv_{A}$ is denoted by $\operatorname{nec}\left(\equiv_{A}\right)$. 
A full binary tree is a rooted binary tree in which every non-leaf node has two children. Let $L(T)$ be a set of leaves in a tree $T$. A decomposition tree of a graph $G$ is a pair $(T, \delta)$ of a full binary tree $T$ with $|V(G)|$ leaves and a bijection $\delta: L(T) \rightarrow V(G)$. For a node $a$ in $T$, let $T_{a}$ be the subtree of $T$ rooted at $a$, and let

$$
A_{a} \stackrel{\text { def }}{=}\left\{v \in V(G) \mid \delta^{-1}(v) \in L\left(T_{a}\right)\right\} .
$$

A cut $\left(A_{a}, \overline{A_{a}}\right)$ is called a cut of $a$. The boolean-width of a decomposition tree $(T, \delta)$, denoted by $\operatorname{boolw}(T, \delta)$, is the maximum $\log \operatorname{nec}\left(\equiv_{A_{a}}\right)$ over all nodes $a$ in $T$. The booleanwidth of $G$ is the minimum boolw $(T, \delta)$ over all decomposition trees $(T, \delta)$ of $G$.

The followings are shown in [12], [13].

Theorem III. The weighted dominating set problem can be solved in $O\left(n^{2}+n k 2^{3 k}\right)$ time provided that a decomposition tree of boolean-width $k$ is given.

Theorem IV. The number of dominating sets of a fixed size can be computed in $O\left(n^{3} k 2^{3 k}\right)$ time provided that a decomposition tree of boolean-width $k$ is given.

A decomposition tree of boolean-width $O(\log n)$ can be obtained in polynomial time for some intersection graphs, such as permutation graphs and interval bigraphs [4], [5], [35]. It follows from Theorems III and IV that the weighted dominating set problem and the dominating set counting problem can be solved in polynomial time for these graphs.

It is pointed out in [56] that the boolean-width of any ORG is $O(\log n)$. The following shows that the booleanwidth of a $2-\mathrm{DORG}$ is indeed at $\operatorname{most} \log n$.

Theorem 3. A decomposition tree of boolean-width at most $\log n$ can be obtained in $O\left(n^{2}\right)$ time for 2-DORGs.

To prove the theorem, we follow the arguments used in [4], [5]. Let $(T, \delta)$ be a decomposition tree of a graph $G$. For a node $a$ in $T$, we define that

$$
r_{a} \stackrel{\text { def }}{=} \max _{S \subseteq A_{a}} \min _{S^{\prime} \subseteq S}\left\{\left|S^{\prime}\right| \mid S^{\prime} \equiv_{A_{a}} S\right\} .
$$

Also, for a decomposition tree $(T, \delta)$ of $G$, we define that $r_{(T, \delta)}$ is the maximum $r_{a}$ over all nodes $a$ in $T$. The following is shown in [4].

Theorem V. $\operatorname{boolw}(T, \delta) \leq r_{(T, \delta)} \cdot \log n$.

As the full binary tree of the decomposition tree of a graph $G$, a caterpillar is used in [4], [5]. We use a special kind of caterpillar as follows. Let $\left(w_{1}, w_{2}, \ldots, w_{n}\right)$ be a total ordering of the vertices of $G$. Let $T$ be a caterpillar obtained from a path $\left(a_{1}, a_{2}, \ldots, a_{n}\right)$ attaching a leaf $l_{i}$ to $a_{i}$ for each $i, 2 \leq i \leq n$, and let $a_{n}$ be a root of $T$. Let $\delta$ be a bijection from $L(T)$ to $V(G)$ such that $a_{1}$ mapped to $w_{1}$ and $l_{i}$ mapped to $w_{i}$ for each $i, 2 \leq i \leq n$. The pair $(T, \delta)$ is called the decomposition caterpillar of $G$. Notice that $A_{a_{i}}=\left\{w_{j} \in\right.$ $V(G) \mid j \leq i\}$ for any node $a_{i}$ in $T$.
Proof of Theorem 3. Let $G$ be a 2-DORG with bipartition $(U, V)$ and a point representation $\mathcal{P}(G)=\left\{\left(x_{w}, y_{w}\right) \mid w \in\right.$ $V(G)\}$. We assume without loss of generality that every $x_{w}$ is distinct and every $y_{w}$ is distinct [52]. We refer to $x_{w}$ and $y_{w}$ as the $x$ - and $y$-coordinate of $w$, respectively.

Let $\left(w_{1}, w_{2}, \ldots, w_{n}\right)$ be the increasing order of $x$ coordinates of vertices of $G$, that is, the total ordering of $V(G)$ such that for any $w_{i}$ and $w_{j}, i<j$ if and only if $x_{w_{i}}<x_{w_{j}}$. Let $(T, \delta)$ be a decomposition caterpillar obtained from such ordering. For any leaf $l$ in $T, r_{l}=1$ since $\left|A_{l}\right|=1$. We show that for any internal node $a_{i}$ in $T, r_{a_{i}}=1$. For each $S \subseteq A_{a_{i}}=\left\{w_{j} \in V(G) \mid j \leq i\right\}$, let $u_{S} \in S \cap U$ be a vertex with the minimum $y$-coordinate over all vertices in $S \cap U$. Notice that $N(S) \cap \overline{A_{a_{i}}} \subseteq \overline{A_{a_{i}}} \cap V$ since no vertices in $\overline{A_{a_{i}}} \cap U$ are adjacent to a vertex in $A_{a_{i}}$. Notice also that any vertex $v \in \overline{A_{a_{i}}} \cap V$ adjacent to a vertex $u \in S \cap U$ is also adjacent to $u_{S}$ since $x_{u_{S}}<x_{v}$ and $y_{u_{S}}<y_{u}<y_{v}$. Therefore, we have $S \equiv_{A_{a_{i}}}\left\{u_{S}\right\}$ for any $S \subseteq A_{a_{i}}$, and $r_{a_{i}}=1$. Thus, $r_{(T, \delta)}=1$, and the boolean-width of the decomposition caterpillar is at most $\log n$ by Theorem V.

Since the representation of a 2-DORG can be obtained in $O\left(n^{2}\right)$ time [50], and a decomposition caterpillar can be obtained by sorting the points in the $x y$-plane, the overall time complexity is $O\left(n^{2}\right)$.

We have the following from Theorems III and 3.

Theorem 4. The weighted dominating set problem can be solved in $O\left(n^{4} \log n\right)$ time for 2-DORGs.

We also have the following from Theorems IV and 3.

Theorem 5. The number of dominating sets in a 2-DORG of a fixed size can be computed in $O\left(n^{6} \log n\right)$ time.

\section{Induced Matchings and Strong Edge Colorings}

It has been known that the induced matching problem for a graph $G$ can be reduced to the independent set problem for the square of line graph of $G$ [15]. Indeed, the induced matching problem for many classes of graphs can be solved in this way [9], [15]-[18], [31], [32], [38]. The square of line graph of $G$ is a graph $L^{2}(G)$ such that $V\left(L^{2}(G)\right)=E(G)$ and for any $e, f \in V\left(L^{2}(G)\right),(e, f) \in E\left(L^{2}(G)\right)$ if and only if $e$ and $f$ share a common endvertex or they are joined by an edge in $G$. We can see that any induced matching of $G$ corresponds to the independent set of $L^{2}(G)$ and vice versa, and any strong edge coloring of $G$ corresponds to the coloring of $L^{2}(G)$ and vice versa. It is also known that a chain subgraph cover of any chordal bipartite graph (and thus, of any 2-DORG) $G$ corresponds to the clique cover of $L^{2}(G)$ [1].

\subsection{2-Directional Orthogonal Ray Graphs}

Lemma 6. If $G$ is a 2-DORG, $L^{2}(G)$ is a trapezoid graph. Moreover, a box representation of $L^{2}(G)$ can be obtained in $O(m)$ time from the orthogonal ray representation of $G$. 


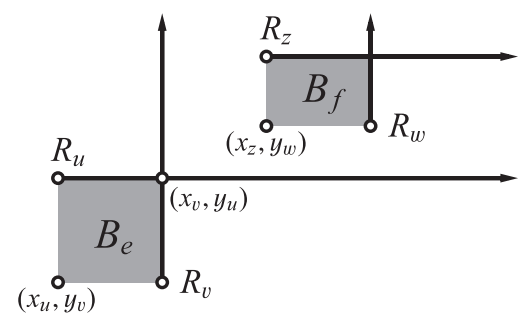

Fig. 3 The box representation of $L^{2}(G)$ constructed from the orthogonal ray representation of $G$.

Proof. Let $G$ be a 2-DORG with bipartition $(U, V)$ and an orthogonal ray representation $\mathcal{R}(G)=\left\{R_{w} \mid w \in V(G)\right\}$. We assume without loss of generality that every $R_{u}, u \in U$, is a rightward ray and every $R_{v}, v \in V$, is an upward ray. Let $\left(x_{w}, y_{w}\right)$ be the endpoint of $R_{w} \in \mathcal{R}(G)$. We further assume that every $x_{w}$ is distinct and every $y_{w}$ is distinct [52]. Notice that for any $u \in U$ and $v \in V,(u, v) \in E(G)$ if and only if $x_{u}<$ $x_{v}$ and $y_{u}>y_{v}$. For each edge $e=(u, v) \in E(G)$ with $u \in U$ and $v \in V$, let $B_{e}$ be a box in the $x y$-plane whose upper left corner point is $\left(x_{u}, y_{u}\right)$ and lower right corner point is $\left(x_{v}, y_{v}\right)$ (See Fig. 3 for example). Notice that $\operatorname{ur}\left(B_{e}\right)=\left(x_{v}, y_{u}\right)$ and $l l\left(B_{e}\right)=\left(x_{u}, y_{v}\right)$. Let $H$ be a trapezoid graph with $V(H)=$ $E(G)$ and the box representation $\mathcal{B}(H)=\left\{B_{e} \mid e \in E(G)\right\}$. Since for any $e=(u, v), f=(z, w) \in E(G)$ with $u, z \in U$ and $v, w \in V,(e, f) \notin E(H) \Leftrightarrow B_{e}<\mathbb{R}^{2} B_{f}\left[\begin{array}{ll}\text { or } B_{f}<\mathbb{R}^{2} & B_{e}\end{array}\right]$ $\Leftrightarrow\left(x_{v}<x_{z}\right) \wedge\left(y_{u}<y_{w}\right)\left[\right.$ or $\left.\left(x_{w}<x_{u}\right) \wedge\left(y_{z}<y_{v}\right)\right] \Leftrightarrow$ $((z, v) \notin E(G)) \wedge((u, w) \notin E(G)) \Leftrightarrow(e, f) \notin E\left(L^{2}(G)\right)$, we conclude that $H=L^{2}(G)$. Thus, $L^{2}(G)$ is a trapezoid graph.

Since the above procedure requires constant time for each edge in $G$, the box representation of $L^{2}(G)$ can be obtained in $O(m)$ time from the orthogonal ray representation of $G$.

Recall that for a 2-DORG $G$, the induced matching, the strong edge coloring, and the chain subgraph cover correspond to the independent set, the coloring, and the clique cover of $L^{2}(G)$. It is shown in [26] that the weighted independent set problem, the coloring problem, and the clique cover problem can be solved in $O(n \log n)$ time for trapezoid graphs provided that box representations are given. Since $\left|L^{2}(G)\right|=|E(G)|$, we have from Lemma 6 that the corresponding problems can be solved in $O(m \log m)$ time for 2-DORGs. Notice that $O(m \log m)=O(m \log n)$ since $m=O\left(n^{2}\right)$. Since an orthogonal ray representation of a 2-DORG can be obtained in $O\left(n^{2}\right)$ time [50], we have the following.

Theorem 7. The weighted induced matching problem, the strong edge coloring problem, and the chain subgraph cover problem can be solved in $O\left(n^{2}+m \log n\right)$ time for 2-DORGs. Moreover, these problems can be solved in $O(m \log n)$ time for 2-DORGs provided that orthogonal ray representations are given.

We note that the necessary condition in Lemma 6 is not sufficient, since $C_{6}$, a cycle of length 6 , is a counterexample: We can see from Theorem II that $C_{6}$ is not a 2-DORG, whereas since $\overline{L^{2}\left(C_{6}\right)}=3 K_{2}$ (three disjoint edges), $\overline{L^{2}\left(C_{6}\right)}$ is a 2-DORG, which implies from Theorem 1 (vi) that $L^{2}\left(C_{6}\right)$ is a trapezoid graph.

\subsection{3-Directional Orthogonal Ray Graphs}

We first show a necessary condition of 3-DORGs. To prove the condition, we use the following.

Lemma 8. For any permutation $\pi$ on $\{0,1, \ldots, 2 k\}, k \geq 1$, there exists $i, 0 \leq i \leq 2 k$, such that $\pi_{i}<\pi_{i+k+1}<\pi_{i+1}$ or $\pi_{i}>\pi_{i+k+1}>\pi_{i+1}$ (Subscripts are modulo $2 k+1$ ).

Proof. It suffices to show that there exists $i, 0 \leq i \leq 2 k$, such that $\left(\pi_{i}-\pi_{i+k+1}\right)\left(\pi_{i+k+1}-\pi_{i+1}\right)>0$. Suppose contrary that $\left(\pi_{i}-\pi_{i+k+1}\right)\left(\pi_{i+k+1}-\pi_{i+1}\right)<0$ for any $i, 0 \leq i \leq 2 k$. Then, we have

$$
\prod_{i=0}^{2 k}\left(\pi_{i}-\pi_{i+k+1}\right)\left(\pi_{i+k+1}-\pi_{i+1}\right)=\prod_{i=0}^{2 k}\left(\pi_{i}-\pi_{i+k+1}\right)^{2}<0,
$$

a contradiction.

We have the following from Lemma 8 .

Theorem 9. Any 3-DORG contains no edge-asteroids.

Proof. Let $G$ be a 3-DORG with bipartition $(U, V)$ and an orthogonal ray representation $\mathcal{R}(G)=\left\{R_{w} \mid w \in V(G)\right\}$. We assume without loss of generality that every $R_{v}, v \in V$, is an upward ray. Let $\left(x_{w}, y_{w}\right)$ be the endpoint of $R_{w} \in \mathcal{R}(G)$, and we have that every $x_{v}, v \in V$, is distinct since the vertical rays in $\mathcal{R}(G)$ are disjoint. We prove the theorem by contradiction. Suppose contrary that $G$ has an edge-asteroid $\left(e_{0}, e_{1}, \ldots, e_{2 k}\right)$ of size $2 k+1, k \geq 1$, where $e_{i}=\left(u_{i}, v_{i}\right)$ with $u_{i} \in U$ and $v_{i} \in V$ for any $i, 0 \leq i \leq 2 k$. Let $\pi$ be a permutation on $\{0,1, \ldots, 2 k\}$ such that $\pi_{i}<\pi_{j}$ if and only if $x_{v_{i}} \leq x_{v_{j}}$ (Recall that two edges in an edge-asteroid may share a common endvertex as shown in Fig. 1). We assume that subscript additions are modulo $2 k+1$.

Now, we have from Lemma 8 that there exists a positive integer $i$ such that $\pi_{i}<\pi_{i+k+1}<\pi_{i+1}$ or $\pi_{i}>\pi_{i+k+1}>\pi_{i+1}$. We have that $e_{i}$ and $e_{i+k+1}$ share no common endvertex, and $e_{i+1}$ and $e_{i+k+1}$ share no common endvertex by the definition of edge-asteroids. Two rays $R_{u_{i+k+1}}$ and $R_{v_{i+k+1}}$ divide the plane into two regions, and $R_{v_{i}}$ and $R_{v_{i+1}}$ are in the different region since $R_{v_{i}}$ and $R_{v_{i+1}}$ are upward rays. Hence, any path from $e_{i}$ to $e_{i+1}$ must have a vertex adjacent to $u_{i+k+1}$ or $v_{i+k+1}$, contradicting to the definition of edge-asteroid. Thus, $G$ contains no edge-asteroids.

Now, we consider the graphs that contain no edgeasteroids. For a set of vertices $A \subseteq V(G)$ of a graph $G$, we use $G-A$ to denote the graph obtained from $G$ by deleting all vertices in $A$. We have the following.

Lemma 10. For any edge e in a graph $G$,

$$
L^{2}\left(G-N_{G}[e]\right)=L^{2}(G)-N_{L^{2}(G)}[e] .
$$


Proof. Since $N_{L^{2}(G)}[e]$ is the set of edges of $G$ incident to a vertex of $G$ in $N_{G}[e]$, we have that $V\left(L^{2}\left(G-N_{G}[e]\right)\right)=$ $E\left(G-N_{G}[e]\right)=E(G) \backslash N_{L^{2}(G)}[e]=V\left(L^{2}(G)-N_{L^{2}(G)}[e]\right)$. For any $f, g \in V\left(L^{2}\left(G-N_{G}[e]\right)\right),(f, g) \in E\left(L^{2}\left(G-N_{G}[e]\right)\right)$ if and only if $f$ and $g$ share a common vertex in $G$ or there exists an edge $h \in E\left(G-N_{G}[e]\right)$ joining $f$ and $g$. Since such $h$ is incident to no vertex of $G$ in $N_{G}[e]$ and $h \notin N_{L^{2}(G)}[e]$ in $L^{2}(G)$, we have that $(f, g) \in E\left(L^{2}\left(G-N_{G}[e]\right)\right) \Leftrightarrow(f, g) \in E\left(L^{2}(G)\right)$ $\Leftrightarrow(f, g) \in E\left(L^{2}(G)-N_{L^{2}(G)}[e]\right)$. Thus, we conclude that $L^{2}\left(G-N_{G}[e]\right)=L^{2}(G)-N_{L^{2}(G)}[e]$.

We have the following from the definition of edgeasteroids and Lemma 10.

Lemma 11. An edge-asteroid in a graph $G$ corresponds to the asteroid in $L^{2}(G)$ and vice versa.

Proof. Notice that a sequence of vertices $\left(v_{0}, v_{1}, \ldots, v_{2 k}\right)$, $k \geq 1$, of $G$ is an asteroid of size $2 k+1$ if and only if for any $i, 0 \leq i \leq 2 k$, two vertices $v_{i}$ and $v_{i+1}$ are in the same connected component of $G-N_{G}\left[v_{i+k+1}\right]$ (Subscripts are modulo $2 k+1)$. Notice also that a sequence of edges $\left(e_{0}, e_{1}, \ldots, e_{2 k}\right)$, $k \geq 1$, of $G$ is an edge-asteroid of size $2 k+1$ if and only if for any $i, 0 \leq i \leq 2 k$, two edges $e_{i}$ and $e_{i+1}$ are in the same connected component of $G-N_{G}\left[e_{i+k+1}\right]$. Thus, we have the lemma from Lemma 10.

We have the following from Theorem I and Lemma 11

Theorem 12. A graph $G$ contains no edge-asteroids if and only if $L^{2}(G)$ is a co-comparability graph.

Recall that any induced matching of $G$ corresponds to the independent set of $L^{2}(G)$, and any strong edge coloring of $G$ corresponds to the coloring of $L^{2}(G)$. For cocomparability graphs, the independent set problem can be solved in linear time [41], and the coloring problem can be solved in $O\left(n^{3}\right)$ time [30]. Since it takes $O\left(\mathrm{~m}^{2}\right)$ time to obtain $L^{2}(G)$ from $G$, we have the following from Theorem 12.

Theorem 13. For the graphs that contain no edgeasteroids, the induced matching problem and the strong edge coloring problem can be solved in $O\left(\mathrm{~m}^{2}\right)$ and $O\left(\mathrm{~m}^{3}\right)$ time, respectively.

The following is shown in [18].

Theorem VI. The weighted induced matching problem can be solved in $\mathrm{O}\left(\mathrm{m}^{4}\right)$ time for graphs that contain no edgeasteroids of size 3 .

We have the following from Theorem 9, 13, and VI.

Corollary 14. For 3-DORGs, the induced matching problem, the weighted induced matching problem, and the strong edge coloring problem can be solved in $O\left(\mathrm{~m}^{2}\right), O\left(\mathrm{~m}^{4}\right)$, and $O\left(\mathrm{~m}^{3}\right)$ time, respectively.

\subsection{Orthogonal Ray Graphs}

A set of vertices $A \subseteq V(G)$ of a graph $G$ is called an asteroidal set [57] if for every $a \in A$, the vertices in $A \backslash\{a\}$ are contained in the same connected component of $G-N[a]$. The maximum cardinality of an asteroidal set in $G$ is called the asteroidal number of $G$.

A set of edges $F \subseteq E(G)$ is called an edge-asteroidal set [18] if for every $e \in F$, the edges in $F \backslash\{e\}$ are contained in the same connected component of $G-N[e]$. We can see that every edge-asteroidal set is an induced matching, and a set of two edges is an edge-asteroidal set if it forms $2 K_{2}$. The maximum cardinality of an edge-asteroidal set in $G$ is called the asteroidal index of $G$. The following is shown in [18].

Theorem VII. The weighted induced matching problem for graphs with asteroidal index at most $s$ can be solved in $O\left(m^{s+2}\right)$ time.

We note that Theorem VII can be derived from Lemma 10, which implies that an edge-asteroidal set in a graph $G$ corresponds to the asteroidal set in $L^{2}(G)$ (This is also proved in [18]). Since the independent set problem for graphs with asteroidal number at most $s$ can be solved in $O\left(n^{s+2}\right)$ time [11], we have the theorem.

We have the following for ORG. It should be noted that the following lemma is implicitly shown in [44], [48].

Lemma 15. The asteroidal index of any ORG is at most 4.

Proof. Let $G$ be an ORG with bipartition $(U, V)$ and an orthogonal ray representation $\mathcal{R}(G)=\left\{R_{w} \mid w \in V(G)\right\}$. Notice that for the representation $\mathcal{R}(G)$, each edge of $G$ can be classified into four types as up-right, down-right, up-left, or down-left, depending on the orientations of the horizontal ray (rightward or leftward) and the vertical ray (upward or downward) corresponding to the endvertices of the edge.

Now, we prove the lemma by contradiction. Suppose contrary that $G$ has an edge-asteroidal set $F$ of size at least 5. Since $|F| \geq 5$, at least two edges in $F$ have the same type. We assume without loss of generality that edges $e$ and $f$ are both of type up-right. For an edge $(u, v) \in E(G)$ of type up-right with $u \in U$ and $v \in V$, two rays $R_{u}$ and $R_{v}$ divide the plane into two regions, and we refer to the region above $R_{u}$ and to the right of $R_{v}$ as the inner region of $(u, v)$, and the other as the outer region. Since no pairs of edges in $F$ are joined by an edge, we can further assume that the rays corresponding to the endvertices of $e$ lie in the inner region of $f$, and we have that the rays corresponding to the endvertices of the other edges in $F$ lie in the outer region of $f$. Hence, any path from $e$ to any other edge in $F$ must have a vertex adjacent to at least one of the endvertices of $f$, contradicting to the definition of edge-asteroidal set. Thus, $G$ contains no edge-asteroidal sets of size at least 5 .

Thus, we have the following from Theorem VII and Lemma 15.

Theorem 16. The weighted induced matching problem can be solved in $O\left(\mathrm{~m}^{6}\right)$ time for ORGs. 


\section{Concluding Remarks}

It should be noted that Theorem 3 indicates that the number of independent sets in a 2-DORG of a fixed size can be computed in $O\left(\alpha^{2} n^{4} \log n\right)$ time, where $\alpha$ is the size of the maximum independent set of the graph [13]. Theorem 3 also implies that several optimization problems can be solved in polynomial time for 2-DORGs (See [13], [14] for details).

It is shown in [13], [14] that the dominating set problem can be solved in polynomial time for the graph that has a decomposition tree of boolean-width $O(\log n)$. Since such a decomposition tree of any ORG can be obtained from the orthogonal ray representation [56], the problem can be solved in polynomial time for ORGs provided that orthogonal ray representations are given. However, since the complexity of the recognition problem for ORGs and 3-DORGs remains open, the complexity of the dominating set problem for ORGs and 3-DORGs still remains open.

Recognition of ORGs and 3-DORGs is an interesting open problem. It should be noted that the recognition problems can be solved in polynomial time provided that orientations of rays corresponding to the vertices are given [25]. Also, the problems can be solved in linear time for the case of trees [44].

We also note that the complexity of the strong edge coloring problem for ORGs remains open.

\section{Acknowledgements}

The authors are grateful to Professors Hell, Huang, Otach, and Uehara for careful reading and helpful comments on a preliminary version of the paper. We are also grateful to anonymous referees for careful reading and helpful comments.

\section{References}

[1] A. Abueida, A.H. Busch, and R. Sritharan, "A min-max property of chordal bipartite graphs with applications," Graphs and Combinatorics, vol.26, pp.301-313, 2010.

[2] H. Balakrishnan, C. Barrett, V. Kumar, M. Marathe, and S. Thite, "The distance-2 matching problem and its relationship to the MAClayer capacity of ad hoc wireless networks," IEEE J. Selected Areas Commun., vol.22, pp.1069-1079, 2004.

[3] C.L. Barrett, V.S.A. Kumar, M.V. Marathe, S. Thite, and G. Istrate, "Strong edge coloring for channel assignment in wireless radio networks," Proceedings of the 4th Annual IEEE International Conference on Pervasive Computing and Communications Workshops, pp.106-110, 2006.

[4] R. Belmonte and M. Vatshelle, "Graph classes with structured neighborhoods and algorithmic applications," Proceedings of the 37th international conference on Graph-Theoretic Concepts in Computer Science, Lecture Notes in Computer Science, vol.6986, pp.47-58, 2011.

[5] R. Belmonte and M. Vatshelle, "Graph classes with structured neighborhoods and algorithmic applications," Theoretical Computer Science, vol.511, pp.54-65, 2013

[6] K.P. Bogart, P.C. Fishburn, G. Isaak, and L. Langley, "Proper and unit tolerance graphs," Discrete Applied Mathematics, vol.60, pp.99-117, 1995.
[7] V. Bonifaci, P. Korteweg, A. Marchetti-Spaccamela, and L. Stougie, "Minimizing flow time in the wireless gathering problem," ACM Transactions on Algorithms, vol.7, pp.33:1-33:20, 2011.

[8] A. Brandstädt, E.M. Eschen, and R. Sritharan, "The induced matching and chain subgraph cover problems for convex bipartite graphs," Theoretical Computer Science, vol.381, pp.260-265, 2007.

[9] A. Brandstädt and C.T. Hoàng, "Maximum induced matchings for chordal graphs in linear time," Algorithmica, vol.52, pp.440-447, 2008.

[10] A. Brandstädt, V.B. Le, and J.P. Spinrad, Graph Classes: A Survey, Society for Industrial and Applied Mathematics, Philadelphia, PA, USA, 1999.

[11] H. Broersma, T. Kloks, D. Kratsch, and H. Müller, "Independent sets in asteroidal triple-free graphs," SIAM Journal on Discrete Mathematics, vol.12, pp.276-287, 1999.

[12] B.-M. Bui-Xuan, J.A. Telle, and M. Vatshelle, "Boolean-width of graphs," Proceedings of the 4th International Workshop on Parameterized and Exact Computation (IWPEC 2009), Lecture Notes in Computer Science, vol.5917, pp.61-74, 2009.

[13] B.-M. Bui-Xuan, J.A. Telle, and M. Vatshelle, "Boolean-width of graphs," Theoretical Computer Science, vol.412, pp.5187-5204, 2011.

[14] B.-M. Bui-Xuan, J.A. Telle, and M. Vatshelle, "Fast dynamic programming for locally checkable vertex subset and vertex partitioning problems," Theoretical Computer Science, vol.511, pp.66-76, 2013.

[15] K. Cameron, "Induced matchings," Discrete Applied Mathematics, vol.24, pp.97-102, 1989.

[16] K. Cameron, "Induced matchings in intersection graphs," Discrete Mathematics, vol.278, pp.1-9, 2004.

[17] K. Cameron, R. Sritharan, and Y. Tang, "Finding a maximum induced matching in weakly chordal graphs," Discrete Mathematics, vol.266, pp.133-142, 2003.

[18] J.-M. Chang, "Induced matchings in asteroidal triple-free graphs," Discrete Applied Mathematics, vol.132, pp.67-78, 2003.

[19] H.S. Chao, F.-R. Hsu, and R.C.T. Lee, "An optimal algorithm for finding the minimum cardinality dominating set on permutation graphs," Discrete Applied Mathematics, vol.102, pp.159-173, 2000.

[20] I. Dagan, M.C. Golumbic, and R.Y. Pinter, "Trapezoid graphs and their coloring," Discrete Applied Mathematics, vol.21, pp.35-46, 1988.

[21] P. Damaschke, H. Müller, and D. Kratsch, "Domination in convex and chordal bipartite graphs," Information Processing Letters, vol.36, pp.231-236, 1990.

[22] A. Ershadi, List homomorphisms and bipartite co-circular arc graphs, Master's thesis, Simon Fraser University, 2012.

[23] T. Feder, P. Hell, and J. Huang, "List homomorphisms and circular arc graphs," Combinatorica, vol.19, pp.487-505, 1999.

[24] S. Felsner, "Tolerance graphs and orders," Journal of Graph Theory, vol.28, pp.129-140, 1998.

[25] S. Felsner, G.B. Mertzios, and I. Mustață, "On the recognition of four-directional orthogonal ray graphs," Proceedings of the 38th International Symposium on Mathematical Foundations of Computer Science, Lecture Notes in Computer Science, vol.8087, pp.373-384, 2013.

[26] S. Felsner, R. Müller, and L. Wernisch, "Trapezoid graphs and generalizations, geometry and algorithms," Discrete Applied Mathematics, vol.74, pp.13-32, 1997.

[27] T. Gallai, "Transitiv orientierbare graphen," Acta Mathematica Academiae Scientiarum Hungarica, vol.18, pp.25-66, 1967.

[28] B. Gamble, W.R. Pulleyblank, B. Reed, and F.B. Shepherd, "Right angle free subsets in the plane," Graphs and Combinatorics, vol.11, pp.121-129, 1995.

[29] M.R. Garey and D.S. Johnson, Computers and Intractability: A Guide to the Theroy of NP-Completeness, W.H. Freeman and Company, 1979.

[30] M.C. Golumbic, Algorithmic graph theory and perfect graphs, in vol.57 of Annals of Discrete Mathematics, 2 ed., Elsevier, 2004. 
[31] M.C. Golumbic and R. Laskar, "Irredundancy in circular arc graphs," Discrete Applied Mathematics, vol.44, pp.79-89, 1993.

[32] M.C. Golumbic and M. Lewenstein, "New results on induced matchings," Discrete Applied Mathematics, vol.101, pp.157-165, 2000

[33] P.L. Hammer, U.N. Peled, and X. Sun, "Difference graphs," Discrete Applied Mathematics, vol.28, pp.35-44, 1990.

[34] J. Huang, "Representation characterizations of chordal bipartite graphs," Journal of Combinatorial Theory Series B, vol.96, pp.673683,2006

[35] J.M. Keil, "The dominating set problem in interval bigraphs, abstract," Proceedings of the 3rd Annual Workshop on Algorithmic Graph Theory, p.1, 2012.

[36] J.M. Keil and P. Belleville, "Dominating the complements of bounded tolerance graphs and the complements of trapezoid graphs," Discrete Applied Mathematics, vol.140, pp.73-89, 2004.

[37] S. Kijima, Y. Okamoto, and T. Uno, "Dominating set counting in graph classes," Proceedings of the 17th Annual International Computing and Combinatorics Conference, Lecture Notes in Computer Science, vol.6842, pp.13-24, 2011.

[38] C.M. Krishnamurthy and R. Sritharan, "Maximum induced matching problem on hhd-free graphs," Discrete Applied Mathematics, vol.160, pp.224-230, 2012.

[39] T.-H. Ma and J. Spinrad, "On the 2-chain subgraph cover and related problems," Journal of Algorithms, vol.17, pp.251-268, 1994.

[40] M. Mahdian, "On the computational complexity of strong edge coloring," Discrete Applied Mathematics, vol.118, pp.239-248, 2002.

[41] R.M. McConnell and J. Spinrad, "Modular decomposition and transitive orientation," Discrete Mathematics, vol.201, pp.189-241, 1999.

[42] N. Milosavljević, "On complexity of wireless gathering problems on unit-disk graphs," Ad-hoc, Mobile, and Wireless Networks, Lecture Notes in Computer Science, vol.6811, pp.308-321, 2011.

[43] H. Müller and A. Brandstädt, "The NP-completeness of Steiner tree and dominating set for chordal bipartite graphs," Theoretical Computer Science, vol.53, pp.257-265, 1987.

[44] I. Mustață, K. Nishikawa, A. Takaoka, S. Tayu, and S. Ueno, On orthogonal ray trees. in preparation.

[45] I. Mustață, M. Pergel, A. Takaoka, S. Tayu, and S. Ueno, "On unit grid intersection graphs," Submitted.

[46] C.G. Plaxton, "Vertex-weighted matching in two-directional orthogonal ray graphs," Proceedings of the 24th International Symposium on Algorithms and Computation, Lecture Notes in Computer Science, vol.8283, pp.524-534, 2013.

[47] C.J. Rhee, Y.D. Liang, S.K. Dhall, and S. Lakshmivarahan, "An $O(N+M)$-time algorithm for finding a minimum-weight dominating set in a permutation graph," SIAM Journal on Computing, vol.25, pp.404-419, 1996.

[48] A.M.S. Shrestha, Study of orthogonal ray graphs with applications to nano-circuit design, $\mathrm{PhD}$ thesis, Department of Communications and Integrated Systems, Tokyo Institute of Technology, 2011.

[49] A.M.S. Shrestha, A. Takaoka, S. Tayu, and S. Ueno, "On two problems of nano-PLA design,” IEICE Trans. Inf. \& Syst., vol.E94-D, no.1, pp.35-41, Jan. 2011.

[50] A.M.S. Shrestha, S. Tayu, and S. Ueno, "On orthogonal ray graphs," Discrete Applied Mathematics, vol.158, pp.1650-1659, 2010.

[51] J.A. Soto, Contributions on secretary problems, independent sets of rectangles and related problems, $\mathrm{PhD}$ thesis, Massachusetts Institute of Technology, 2011.

[52] J.A. Soto and C. Telha, "Jump number of two-directional orthogonal ray graphs," Proceedings of the 15th International Conference on Integer Programming and Combinatorial Optimization, Lecture Notes in Computer Science, vol.6655, pp.389-403, 2011.

[53] J.P. Spinrad, "Efficient graph representations," in vol.19 of Fields Institute monographs, American Mathematical Society, 2003.

[54] L.J. Stockmeyer and V.V. Vazirani, "NP-completeness of some generalizations of the maximum matching problem," Information Processing Letters, vol.15, pp.14-19, 1982.
[55] W.T. Trotter and J.I. Moore, "Characterization problems for graphs, partially ordered sets, lattices, and families of sets," Discrete Mathematics, vol.16, pp.361-381, 1976.

[56] M. Vatshelle, Personal communication, 2013.

[57] J.R. Walter, "Representations of chordal graphs as subtrees of a tree," Journal of Graph Theory, vol.2, pp.265-267, 1978.

[58] M. Yannakakis, "The complexity of the partial order dimension problem," SIAM Journal on Algebraic and Discrete Methods, vol.3, pp.351-358, 1982.

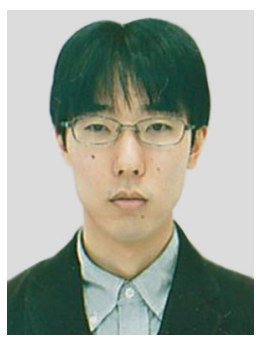

Asahi Takaoka received his B.E. degree in computer science and M.E. degree in communications and integrated systems from Tokyo Institute of Technology, Tokyo, Japan, in 2010 and 2012, respectively. He is currently working towards his doctoral degree at Tokyo Institute of Technology. He is interested in algorithmic graph theory and its applications. He is a student member of IEEE and IEICE.

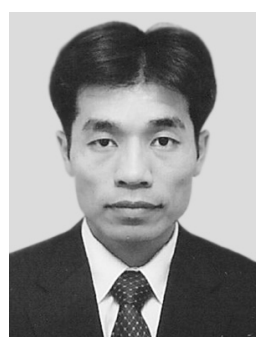

Satoshi Tayu received the B.E., M.E., and D.E. degrees in electrical and electronic engineering from Tokyo Institute of Technology, Tokyo, Japan, in 1992, 1994, and 1997, respectively. From 1997 to 2003, he was a research associate in the School of Information Science, Japan Advanced Institute of Science and Technology, Ishikawa, Japan. He is currently an assistant professor in the Department of Communications and Computer Engineering, Graduate School of Science and Engineering, Tokyo Institute of Technology. His research interests are in parallel computation. $\mathrm{He}$ is a member of IPSJ.

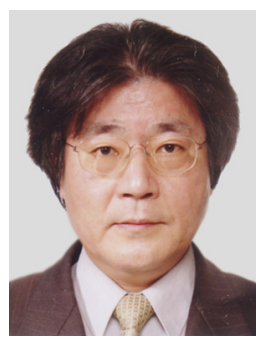

Shuichi Ueno received the B.E. degree in electronic engineering from Yamanashi University, Yamanashi, Japan, in 1976, and M.E. and D.E. degrees in electronic engineering from Tokyo Institute of Technology, Tokyo, Japan, in 1978 and 1982, respectively. Since 1982, he has been with Tokyo Institute of Technology, where he is now a professor in the Department of Communications and Computer Engineering, Graduate School of Science and Engineering. His research interests are in the theory of parallel and VLSI computation. He received the best paper award from the Institute of Electronics and Communication Engineers of Japan in 1986, the 30th anniversary best paper award from the Information Processing Society of Japan in 1990, and the best paper award of APCCAS 2000 from IEEE in 2000. Dr Ueno is a Fellow of IEICE, and a member of IEEE, SIAM, and IPSJ. 